\title{
Transfer of memory retrieval cues in rats
}

\author{
JAMES F. BRiggs, Kelly I. Fitz, AND DAVID C. Riccio \\ Kent State University, Kent, Ohio
}

\begin{abstract}
Two experiments using rats were conducted to determine whether the retrieval of a memory could be brought under the control of new contextual cues that had not been present at the time of training. In Experiment 1, rats were trained in one context and then exposed to different contextual cues immediately, $60 \mathrm{~min}$, or $120 \mathrm{~min}$ after training. When tested in the shifted context, rats that had been exposed shortly after training treated the shifted context as if it were the original context. The control that the previously neutral context had over retrieval disappeared with longer posttraining delays, suggesting the importance of an active memory representation during exposure. Experiment 2 replicated the basic finding and demonstrated that the transfer of retrieval cues was specific to the contextual cues present during exposure. These findings with rats are consistent with findings from infant research (see, e.g., Boller \& Rovee-Collier, 1992) that have shown that a neutral context can come to serve as a retrieval cue for an episode experienced elsewhere.
\end{abstract}

Transfer of control over responding has typically focused on Pavlovian instrumental interactions (Domjan, 2003). Solomon and Turner's (1962) classic study provides a pertinent example. Dogs trained in a shuttle box to avoid a light later received, in a different apparatus, Pavlovian fear conditioning in which a tone was paired with a brief but inescapable shock. Subsequently, when returned to the shuttle apparatus, the dogs made avoidance responses to the tone; that is, the control over avoidance had transferred to the Pavlovian cue.

In Solomon and Turner's (1962) study, transfer depended on direct pairing of a signal (tone) with the shock. More recently, there has been growing interest in memory representations and conditioning (see, e.g., Dwyer, Mackintosh, \& Boakes, 1998; Rescorla, 1973, 1974). In these paradigms, the physical cue is absent at the time of a manipulation; thus, any changes in behavior are presumably attributable to a memory representation. By and large, such studies have focused on altering the value of the conditioned stimulus (CS) by virtue of changes in an unconditioned stimulus in the presence of a representation of the $\mathrm{CS}$. In contrast, little attention has been given to the issue from a memory retrieval perspective: Can a neutral stimulus or contextual cue paired with the representation of a learning episode gain retrieval control over responding?

One approach to this issue takes advantage of the observation that performance is commonly impaired when subjects are tested in a context different from that used at training. This context shift effect, found in both human and animal studies (see, e.g., Boller \& Rovee-Collier, 1992; Godden \& Baddeley, 1975; Gordon, McCracken, DessBeech, \& Mowrer, 1981; Smith, 1979; Zhou \& Riccio, 1996), is typically attributed to a memory deficit resulting from the removal of retrieval cues. In one of the few studies focusing on memory transfer, Boller and RoveeCollier (1992) trained 6-month-old infants to kick a leg to activate a mobile and then immediately exposed them to a novel context (crib liner). At testing, the infants responded in the new context as if it were the training context. Thus, the memory was apparently recoded into the context present while the memory was active.

Although the temporal relationship between training and exposure to the new context was not manipulated in that study, later work using a retroactive interference paradigm demonstrated that the effect of exposure decreased with longer delays (Rossi-George \& Rovee-Collier, 1999). This outcome is consistent with the many findings showing that the severity of retrograde amnesia is inversely related to the interval between training and the amnesic insult. The time-dependent gradient of amnesia is often viewed as resulting from disruption of consolidation - that is, a putative storage process but an alternative retrievaloriented view is conceptually more compatible with the Rovee-Collier findings (Hinderliter, Webster, \& Riccio, 1975; Riccio, Millin, \& Gisquet-Verrier, 2003; Riccio, Moody, \& Millin, 2002). According to the model, information continues to be processed for a brief period after learning. During this time, the active memory becomes encoded or associated with the altered internal context induced by the amnesic treatment. Amnesic agents such as electroconvulsive shock and lowered body temperature are stressors that transiently change the internal state or context. The absence of these contextual cues at a later test results in the memory deficit - that is, retrograde amnesia. With longer intervals between the training episode and the amnesic treatment, the memory is more likely to be encoded within the normal context, producing the characteristic temporal gradient of memory impairment.

D. C. Riccio, driccio@kent.edu 
These two lines of research raise the intriguing question of whether a memory representation in animals could become encoded with or transferred to a new environmental context, which would be reflected in that context's functioning as a retrieval cue. Furthermore, would such a transfer of control follow a temporal gradient, declining as memory activity subsided?

\section{EXPERIMENT 1}

Experiment 1 was designed to investigate whether retrieval cues could be transferred to a new context by exposing subjects to the new context immediately following original training, while the memory was presumably still active. To evaluate any transfer, our strategy exploited the tendency for performance to be impaired when the training and test contexts differ - that is, the context shift effect described above. Thus, if retrieval cues become associated with the new context, the context shift effect should be alleviated. Furthermore, since memory activity should subside over time, it is important to determine whether cue transfer would diminish as a function of the length of the interval from conditioning to exposure.

\section{Method \\ Subjects. The subjects were 50 Long-Evans rats, approximately 85 days old. The animals were individually housed and were main- tained on a 15:9-h light:dark cycle. Food and water were available ad lib throughout the course of the experiment. \\ Apparatus and Contexts. Training and testing were conducted in two identical $43.18 \times 17.78 \times 17.78 \mathrm{~cm}$ shuttle boxes with grid floors. Each shuttle box was divided by a guillotine door into two compartments - one black and one white - of equal size. The ex- posure chamber was a $22 \times 22 \times 23.5 \mathrm{~cm}$ box with clear Plexi- glas walls and lid. The chamber was placed near the training/testing shuttle box in each context during exposure. \\ The two shuttle boxes were located in separate rooms that served as contexts. Context A was a $1.62 \times 2.33 \mathrm{~m}$ room with white walls, scented with Airwick Wizard Country Berries air freshener. White noise $(76 \mathrm{~dB})$ was presented at all times in this context. The room was illuminated by a $25-\mathrm{W}$ red light bulb above the shuttle box. Context B was a brightly lit $1.83 \times 2.74 \mathrm{~m}$ room with white walls. Posters were placed on each wall to provide visual cues. This room was illuminated by fluorescent house lights. Context B was not arti- ficially scented, and no white noise was present.}

Procedure. Prior to the beginning of the experiment, all subjects were handled for $2 \mathrm{~min}$ on three consecutive days. Groups of 10 rats were randomly assigned to one of five conditions; all rats received fear conditioning in either Context A or Context B. Assignment to the contexts was counterbalanced in such a way that within each group, 5 rats were trained in Context A and 5 in Context B. For simplicity of exposition, we refer to context shifts generically, regardless of the actual shift (A to B or B to A) used.

At training, the rat was brought into the context on the experimenter's arm and remained there for $15 \mathrm{sec}$ to provide brief exposure to the context. The rat was then placed in the white compartment of the shuttle box facing away from the closed guillotine door. After $15 \mathrm{sec}$, the guillotine door was raised and the latency to cross into the black compartment (all four paws) was recorded. The door was then lowered and two inescapable footshocks $(0.5 \mathrm{~mA}$, lasting $1 \mathrm{sec})$ were delivered $5 \mathrm{sec}$ and $10 \mathrm{sec}$ after the door was lowered. Five seconds after the last footshock, the animal was removed and returned to its home cage (except for the immediately exposed group; see below).

Following training, three shift condition groups-immediate exposure (imm. exp.), 60-minute exposure (60 exp.), and 120-min exposure (120 exp.) - received exposure to the context that differed from training. Exposure consisted of bringing the rat into the shifted context and immediately placing the animal in the white compartment of the shuttle box in an attempt to maintain the activity of the newly acquired information. After $15 \mathrm{sec}$ of exposure to the white side, the rat was removed and placed in the exposure chamber for $4 \mathrm{~min}, 45 \mathrm{sec}$. Following the 5 -min exposure treatment, the rat was returned to its home cage. The imm. exp. group received exposure immediately following training. The 60 exp. and 120 exp. groups received exposure treatment $60 \mathrm{~min}$ and $120 \mathrm{~min}$ after training, respectively.

Two groups did not receive the exposure treatment in the shifted context. These groups were simply tested either in the same context as training (same group) or in the shifted context (shift group). The shift-with-exposure groups (imm. exp., 60 exp., and 120 exp.) were tested in the context different from the training context.

Twenty-four hours after training, all groups received a 10-min passive avoidance test identical to that used in the training trials except that no shocks were delivered and the guillotine door remained open. The rat was placed on the white side and allowed to choose between the white and black compartments. The cross-through latency and total time spent (TTS) on the safe (white) side were recorded as the dependent measures. As the patterns of results were generally similar on both measures, only TTS scores are reported.

\section{Results and Discussion}

Training. Rats in all five groups exhibited short cross latencies at training with group means ranging from $8.8 \mathrm{sec}$ to $11.9 \mathrm{sec}$. An ANOVA performed on cross-through latencies at training revealed no differences among the five groups $[F(4,45)=0.43, p>.50]$.

Counterbalancing. There were no differences among cross-through latencies at training, cross-through latencies at test, and TTS in either context. Accordingly, the contexts were collapsed within each group for all further analyses.

Testing. Figure 1 shows the mean TTS scores for all five groups at test. An ANOVA revealed that the groups differed significantly $[F(4,45)=15.68, p<.001]$. Tukey's HSD post hoc tests were conducted to compare group differences.

As can be seen, a context shift was obtained, with the shift group showing significantly less fear (shorter TTS) than the same group $(p<.001)$. Thus, being trained and tested in a different context impaired performance. The context shift was attenuated by the transfer of contextual cues to the shifted context, since the group that was immediately exposed to the shifted context after training (imm. exp.) displayed as much fear as the same group $(p>.50)$ and significantly more fear than the shift group $(p<.001)$.

The transfer effect was time limited, since it was not evident in the groups with the two longer intervals between conditioning and exposure. Results from both of the delay groups (60 exp. and 120 exp.) differed significantly from those of the imm. exp. group $(p s<.01)$ but did not differ from the shift group's results $(p \mathrm{~s}>.50)$. This finding indicates that mere exposure to the new context, irrespective of memory transfer, cannot account for the reduction of the context shift effect.

In summary, Experiment 1 demonstrated that retrieval cues could be transferred to a new context if an animal was exposed to a different context immediately after train- 


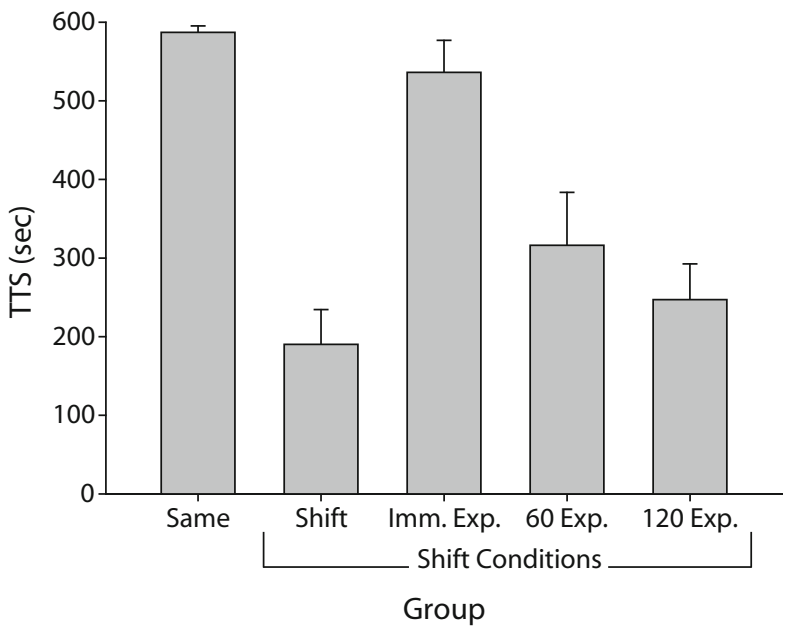

Figure 1. Mean total time spent (TTS), in seconds, on the safe (white) side for all groups in Experiment 1. Error bars represent standard errors of the means. Same and shift groups represent a context shift effect. The imm. exp. group demonstrates that exposure to the shifted context immediately after training alleviates the shift effect. The 60 exp. and 120 exp. groups show that this effect is time limited.

ing. Experiment 1 also showed that with longer trainingto-exposure intervals, the transfer was not effective, an outcome similar to the time dependency effects of retrograde amnesia. Thus, exposure to the novel context while the memory is active presumably allows the information to become associated with that context, but the transfer does not occur with long posttraining delays.

\section{EXPERIMENT 2}

Experiment 1 demonstrated that retrieval cues can be transferred to a new context. However, it is not clear whether the exposure used in Experiment 1 would produce fear in any context or whether the fear was specific to the context in which the exposure took place. Experiment 2 was designed to determine whether the retrieval cues were specific to those cues present at the time of exposure. To avoid the potential contamination of generalization between contexts, we attempted to make a third context, $\mathrm{C}$, distinctly different from the test room, $\mathrm{B}$.

\section{Method}

Subjects. Forty Long-Evans rats, approximately 85 days old, were subjects. The animals were obtained and maintained as in Experiment 1.

Apparatus and Contexts. The two shuttle boxes described in Experiment 1 were used. Contexts A and B were also the same as those used in Experiment 1. A third context, $\mathrm{C}$, was added to this experiment. $\mathrm{C}$ was a $2.33 \times 1.62 \mathrm{~m}$ room with plain white walls. This context was illuminated by a single $15-\mathrm{W}$ bulb located on the floor (approximately $1 \mathrm{~m}$ below the apparatus). The shuttle box was situated on top of a wooden table against one wall of the room. Context $\mathrm{C}$ was not artificially scented, and no white noise was present.

Procedure. All animals were handled, trained, exposed, and tested following the procedures described in Experiment 1. Prior to training, the rats were randomly divided into four groups of 10 . As in
Experiment 1, training conditions were counterbalanced with half of each group assigned to Context $\mathrm{A}$ and the other half to Context $\mathrm{B}$.

Following training, two shift condition groups, the $B$ exposure (B exp.) and the $C$ exposure ( $\mathrm{C}$ exp.) groups, received immediate exposure to a shifted context that differed from that of training. To replicate the finding of retrieval cues transfer, the B exp. group was exposed to Context $\mathrm{B}$. To determine whether the transfer of cues was context specific, the $\mathrm{C}$ exp. group was exposed to the third context (Context C). In each case, rats were placed briefly $(15 \mathrm{sec})$ in the white side of the shuttle box that was located in the test context, then placed in the exposure chamber for the remainder of the 5-min treatment. Context $\mathrm{C}$ was not counterbalanced but was always the different context for the $\mathrm{C}$ exp. group. Two other groups (same and shift) did not receive the exposure treatment to the shifted context.

Twenty-four hours after training, all groups were tested as described in Experiment 1. The two groups that did not receive an exposure treatment were tested in either the same context as training (same) or in the shifted context (shift). Both groups in the shift with exposure conditions (B exp. and C exp.) were tested in Context B.

\section{Results and Discussion}

Training. Rats in all four groups exhibited short cross latencies at training, with group means ranging from 10.7 to $14.6 \mathrm{sec}$. An ANOVA performed on training cross latencies revealed no differences between the four groups $[F(3,36)=1.8, p>.10]$.

TTS. Figure 2 shows the mean TTS scores for all four groups at test. An ANOVA revealed that the groups differed significantly $[F(3,36)=124.44, p<.001]$. Tukey's HSD post hoc tests were conducted to compare group differences.

As Figure 2 shows, a context shift was obtained, with the same group showing significantly more fear (longer TTS) than the shift group $(p<.001)$. As in Experiment 1 , training the rats in one context and testing them in a different one impaired their performance. The B exp. group

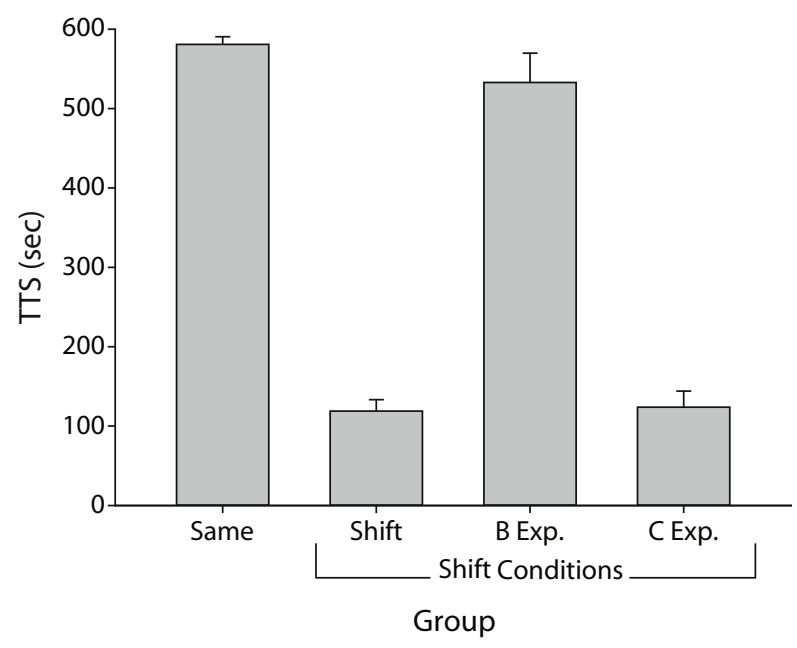

Figure 2. Mean total time spent (TTS), in seconds, on the safe (white) side for all groups in Experiment 2. Error bars represent standard errors of the means. Same and shift groups represent a context shift effect. The B exp. group shows that being exposed to the shifted context immediately after training alleviated the context shift effect. The $\mathrm{C}$ exp. group demonstrates that this effect is context specific. 
replicated the transfer of retrieval cues to a new context. Post hoc tests confirmed this conclusion, with the B exp. group showing as much fear as the same group $(p>.50)$. The rats in the group exposed to a third context (C exp.) but tested in Context B showed significantly less fear than the $\mathrm{B}$ exp. and same groups $(p \mathrm{~s}<.001)$; their level of fear was similar to that of the shift group $(p>.50)$. Thus, it appears that the transfer of retrieval was specific to the context in which exposure occurred.

These data again indicate that rats exposed to a new context shortly after training incorporated the memory from training into the new context's cues, as reflected in the elimination or alleviation of the usual context shift decrement. As this interpretation suggests, the transfer of control over memory is not achieved simply by exposure to any context, since placing rats in a context unrelated to testing produced no improvement in memory. Thus, transfer of retrieval cues is relatively specific to the particular cues present at the time of reencoding exposure.

\section{GENERAL DISCUSSION}

The two experiments presented here examined the possibility that contextual stimuli not present at the time of a learning episode might come to serve as retrieval cues for the target memory. We found that exposing rats to a new context immediately after training alleviated the context shift effect, presumably by integrating the original memory cues with the shifted context cues. The exposure was no longer effective when the interval between conditioning and exposure increased, suggesting that the retrieval cues of the original memory were encoded with the shifted context but that the new retrieval cues had to be present during the active phase of memory encoding in order to become effective. These results, which parallel findings regarding the time-dependent feature of retrograde amnesia, demonstrate the importance of the subject's memory being in an active state (i.e., still processing) in order for the new contextual retrieval cues to become encoded with the memory (see Misanin, Miller, \& Lewis, 1968).

In a second experiment, we replicated the initial finding and demonstrated that the transfer of retrieval cues was specific to those cues present at the time of exposure, since exposure to an irrelevant context following training did not alleviate the context shift effect. Thus, an exposure manipulation per se was not sufficient to enhance retrieval in the novel context, $\mathrm{C}$.

Taken together, these findings confirm and extend the interesting observations initially reported for infants (Boller \& Rovee-Collier, 1992, 1994). Thus, under certain conditions, previously neutral cues can come to control retrieval of memory in rats as well as in infants. Although we favor the notion that an active representation of the target memory becomes associated with or encoded in the new context, other possible mechanisms could be suggested. For example, residual aftereffects of the shock, in conjunction with the white cues, might have mediated associations with the novel context during the brief period that the rats were in the safe compartment before they were placed in the exposure apparatus. However, this form of backward association seems unlikely in view of recent work with a reactivated old memory that did not involve shock around the time of exposure. Using a brief exposure to the conditioned stimulus (a nominal extinction procedure) to induce activity in an old memory, Briggs, Fitz, and Riccio (2006) found a pattern of results similar to that seen here (see also Briggs \& Riccio, in press).

Another possibility is that exposure to the new context while the memory was in an active phase reduced the discriminability of the two contexts. A number of studies have shown that the impaired performance produced by a change in context decreases as a function of the trainingto-test (shift) interval; in short, the two different contexts become more functionally alike (Zhou \& Riccio, 1996; for review, see Riccio, Richardson, \& Ebner, 1984). Very little is known, however, about the mechanisms involved in producing this "forgetting of stimulus attributes," as it has been called. If the alleviation of the context shift found here is based on a rapid loss of discriminability, the paradigm may provide a useful approach for investigating the forgetting of contextual features.

Although these and other important questions about this transfer phenomenon deserve investigation, the major conclusion remains that stimuli never originally associated with an episode can come to serve as retrieval cues for that episode. Just as stimulus generalization can permit fear, for example, to be triggered by a cue not paired with trauma, these transfer studies suggest a mechanism by which neutral contexts can come to control retrieval of an episode experienced elsewhere.

\section{AUTHOR NOTE}

This research was funded by NIMH Grant 37535 to D.C.R. Correspondence concerning this article should be addressed to D. C. Riccio, Department of Psychology, Kent State University, Kent, OH 44242 (e-mail: driccio@kent.edu).

$$
\text { Note-Accepted by David A. Balota's editorial team. }
$$

\section{REFERENCES}

Boller, K., \& Rovee-Collier, C. (1992). Contextual coding and recoding of infants' memories. Journal of Experimental Child Psychology, 53, 1-23.

Boller, K., \& Rovee-Collier, C. (1994). Contextual updating of infants' reactivated memories. Developmental Psychobiology, 27, 241-256.

Briggs, J. F., Fitz, K. I, \& Riccio, D. C. (2006, May). Transfer of old memory retrieval cues. Poster session presented at the annual meeting of the Midwestern Psychological Association, Chicago.

BRIGGS, J. F., \& Riccio, D. C. (in press). Transfer of old "reactivated" memory retrieval cues in rats. Learning \& Motivation.

Domuan, M. (2003). The principles of learning and behavior (5th ed.). Belmont, CA: Thomson/Wadsworth.

Dwyer, D. M., Mackintosh, N. J., \& Boakes, R. A. (1998). Simultaneous activation of the representations of absent cues results in the formation of an excitatory association between them. Journal of Experimental Psychology: Animal Behavior Processes, 24, 163-171.

GodDEN, D. R., \& BADDELEY, A. D. (1975). Context-dependent memory in two natural environments: On land and underwater. British Journal of Psychology, 66, 325-331.

Gordon, W. C., McCracken, K. M., Dess-Beech, N., \& Mowrer, R. R. (1981). Mechanisms for the cueing phenomenon: The addition of the cueing context to the training memory. Learning \& Motivation, 12, 196-211. 
Hinderliter, C. F., Webster, T., \& Riccio, D. C. (1975). Amnesia induced by hypothermia as a function of treatment-test interval and recooling in rats. Animal Learning \& Behavior, 3, 257-263.

Misanin, J. R., Miller, R. R., \& Lewis, D. J. (1968). Retrograde amnesia produced by electroconvulsive shock after reactivation of a consolidated memory trace. Science, 160, 554-555.

RESCORLA, R. A. (1973). Effects of US habituation following conditioning Journal of Comparative \& Physiological Psychology, 82, 137-143.

RESCORLA, R. A. (1974). Effect of inflation of the unconditioned stimulus value following conditioning. Journal of Comparative \& Physiological Psychology, 86, 101-106.

Riccio, D. C., Millin, P. M., \& Gisquet-Verrier, P. (2003). Retrograde amnesia: Forgetting back. Current Directions in Psychological Science, 12, 41-44.

Riccio, D. C., Moody, E. W., \& Millin, P. M. (2002). Reconsolidation reconsidered. Integrative Physiological \& Behavioral Science, 37, 245-253.

Riccio, D. C., Richardson, R., \& Ebner, D. L. (1984). Memory re- trieval deficit based on altered contextual cues: A paradox. Psychological Bulletin, 96, 152-165.

Rossi-George, A., \& Rovee-Collier, C. (1999). Retroactive interference in 3-month-old infants. Developmental Psychobiology, 35, $167-177$.

Smith, S. M. (1979). Remembering in and out of context. Journal of Experimental Psychology: Human Learning \& Memory, 5, 460-471.

Solomon, R. L., \& Turner, L. H. (1962). Discriminative classical conditioning in dogs paralyzed by curare can later control discriminative avoidance responses in the normal state. Psychological Review, 69, 202-219.

ZHou, Y., \& Riccio, D. C. (1996). Manipulation of components of context: The context shift effect and forgetting of stimulus attributes. Learning \& Motivation, 27, 400-407.

(Manuscript received November 17, 2005; revision accepted for publication June 11, 2006.) 